\title{
A statistical jacobian application for power system optimization of voltage stability
}

\author{
Raja Masood Larik ${ }^{1}$, Mohd.Wazir Mustafa ${ }^{2}$, Manoj Kumar Panjwani ${ }^{3}$ \\ ${ }^{1}$ Department of Electrical Engineering NED University of Engineering and Technology Sindh, Pakistan \\ ${ }^{1,2}$ School of Electrical Engineering, Universiti Teknologi Malaysia Skudai, Johor Bharu Malaysia 81310 \\ ${ }^{3}$ Department of Energy Systems Engineering, Sukkur IBA University Pakistan
}

\begin{tabular}{l} 
Article Info \\
\hline Article history: \\
Received Jun 4, 2018 \\
Revised Aug 22, 2018 \\
Accepted Sep 8, 2018 \\
\hline Keywords: \\
Optimal power flow \\
Optimization \\
Power system \\
Voltage stability
\end{tabular}

\begin{abstract}
Despite a tremendous development in optimal power flow (OPF), owing to the obvious complexity, non-linearity and unwieldy size of the large interconnected power systems, several problems remain unanswered in the existing methods of OPF. Seizing specific topics for maximizing voltage stability margin and its implementation, a detailed literature survey discussing the existing methods of solution and their drawbacks are presented in this research. The phenomenon of voltage collapse in power systems, methods to investigate voltage collapse, and methods related to voltage stability are briefly surveyed. Finally, the study presents a statistical method for analyzing a power system through eigenvalue analysis in relation to the singular values of the load flow Jacobian. Future study may focus on changes in theories in conjunction with large power systems.
\end{abstract}

Copyright (C) 2019 Institute of Advanced Engineering and Science. All rights reserved.

\section{Corresponding Author:}

Raja Masood Larik,

Department of Electrical Engineering NED,

University of Engineering and Technology Sindh, Pakistan.

Email: rmlarik@gmail.com

\section{INTRODUCTION}

For the past decades, power system optimization techniques have been subject to many studies for planning and strategy development $[1,2]$. Optimal Power Flow (OPF) refers to a standard term that defines a comprehensive set of obstacles in which researcher pursues achieving one or more objective functions while satisfying constraints dictated by operational and physical restrictions of the electric network. The OPF problems require the determination of the optimal settings of control variables subject to the operating constraints such that the operating objectives are optimized. As a result of the continuous research efforts over the last decades, the OPF algorithms have significantly matured alongside developments in the other areas of technology. Modern OPF algorithms cover both real and reactive power dispatch and can solve very large and complex formulations in a relatively short time. The voltage stability phenomena are discussed in smart grid framework $[3,4]$ and are documented as a significant problem for a sheltered system operation.

As the new concerns like assessment and improvement of voltage stability margin (VSM) bother the power engineers, the OPF algorithms are constantly reviewed and newer methods are evolved in a continuous effort to address these new concerns and better the existing methods. In this orbit of OPF problems, real power scheduling is an integral part. The activities of optimal real power scheduling of a power system assume significance in view of their overbearing financial and operational implications. With increasing fuel costs and declining capital investments, the economics of real power scheduling have a tremendous effect on the profitable and reliable operation of a power system. Proper real power scheduling has a wide-ranging effect on the operation and control of power system. Voltage security and voltage stability of a power system are profoundly affected by real power scheduling. System generation cost and VSM are among many that can 
be improved by effective real power scheduling. Sensor technologies for proper estimation of voltage current and power presented, low voltage ride through LVRT was proposed.

The review of the existing methods for the power system optimization shows that significant progress has been made in the development of reliable and efficient methods. These methods address the problem and use a multitude of formulations and solution techniques. However, there are still some concerns have not been adequately tackled such as considering generation cost minimization and transmission loss minimization along with voltage stability margin. Thus, this study aims to address the issue in accordance with generation cost and transmission loss minimization with respect to voltage stability margin.

This paper organizes as follows. The literature section involves power system overview, optimal real power scheduling, and Scheduling for voltage stability. The next section devotes to OPF optimization techniques and power system classifications. Later in this paper, the power flow Jacobian discussed along with research conclusion and potential research opportunity.

\section{LITERATURE REVIEW}

Power System is a large and complex physical system. It has probably the largest number of interacting elements of varying degrees of complexities. It has enormous capital investment making it one of the costliest physical systems. It spans gigantic geographical expanse making it a physically large system. The power system is a dynamic system. It encompasses generation, transmission, and distribution of electric power to millions of customers delivering billions of joules of energy. Its importance is paramount to the society in all ways. Its dynamic nature and stability are causes for great concern. Kundur in proposed a definition and classification for power system stability as shown in Figure1.

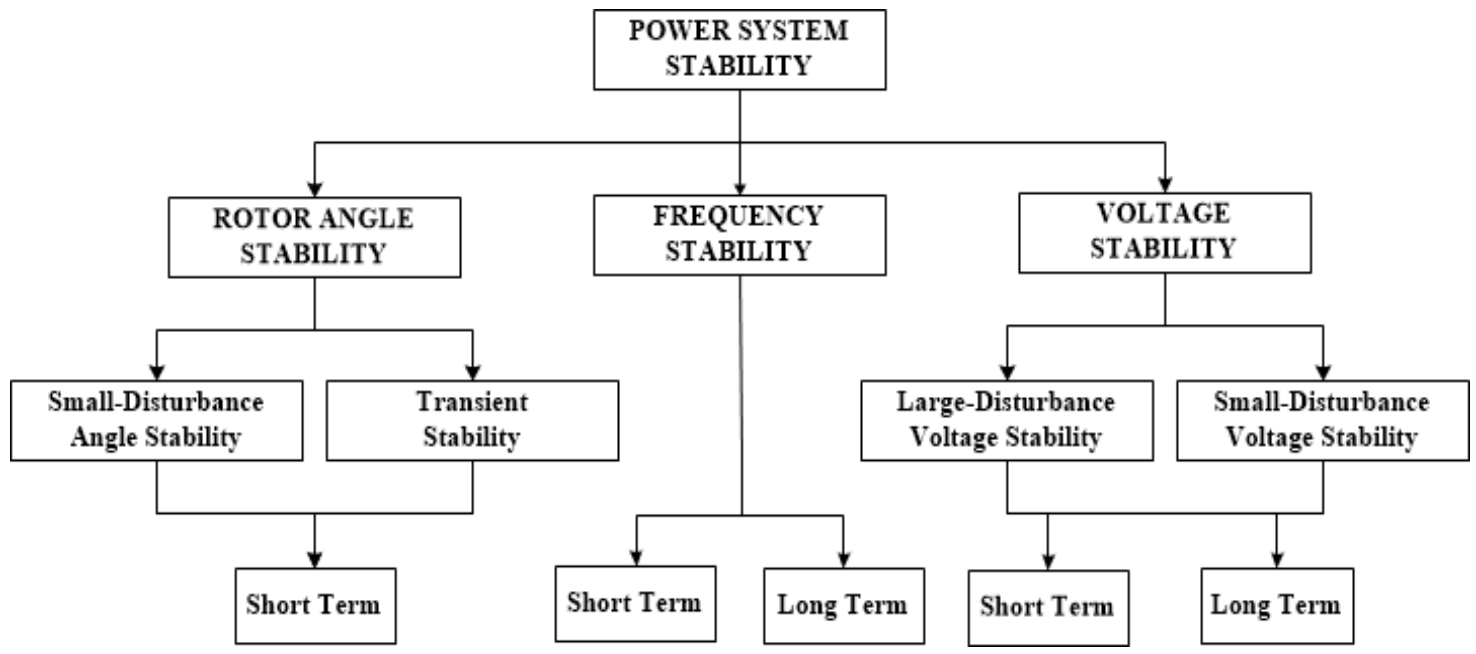

Figure 1. Power system stability

Power system stability is defined as "Power system stability is the ability of an electric power system, for a given initial operating condition, to regain a state of operating equilibrium after being subjected to a physical disturbance, with most system variables bounded so that practically the entire system remains intact."

The mentioned power system stability definition is from the general dynamic system viewpoint. In the above definition, several aspects of power system instability are contained. These aspects are elegantly identified and represented in work. Further, optimal power flow, in essence, deals with generating and routing power through the transmission system to meet chosen objectives and adhere to operating constraints [5]. Some of these constraints are explained here.

\section{OPTIMAL REAL POWER SCHEDULING}

Fuel cost minimization is primarily an operational problem. This is usually referred to as economic dispatch. To minimize the fuel cost, it needs to comprehend the fuel cost curves for all particular power generating components in the system. A precise cost curves illustration may involve a piecewise polynomial 
form or can be estimated through other approaches, with common ones being: 1. Piecewise linear, 2. Quadratic, 3. Cubic, and 4. Piecewise quadratic.

The Linear approximation is not commonly used while the piecewise linear form is used in many production-grade linear programming applications. The quadratic approximation is used in most nonlinear programming applications. Control variables are usually the independent variables in an OPF problem and they include 1. Active power generation, 2. Generator bus voltages, 3. Transformer tap ratios, 4. Phase-shifter angles, and 5. Settings of shunt capacitors and inductors. The application of the aforementioned components as control variables would provide the best (least expensive) result.

There are some assumptions need to be made in modeling of the objectives and constraints where fuel cost curves are smooth and quadratic in nature; only active power generations are controlled for cost minimization and transformer taps, voltage of generators, shunt capacitor settings, and inductor positions are held at their nominal set values throughout the optimization assuming $\mathrm{P}-\mathrm{Q}$ decoupling; current flows are controlled using voltage and phase angle restriction across the lines and finally contingency constraints are neglected.

\section{SCHEDULING USING VOLTAGE STABILITY MARGIN}

Voltage stability assessment and enhancement is a perplexing issue in the field of power systems engineering. It has gained more attention once power systems operations become more complex and many limitations still exist. It has been many years since Angle stability becomes the main issue within the field. Because of deteriorating investments in power facilities and a novel transmission system in the 1980s, the power systems were overloaded, causing phenomena known as voltage instability [6].

Since then, the importance of reactive power in upholding appropriate voltage level throughout the power system become a main concern of engineers. There are some voltage collapse incidents set the initiative to study voltage stability and techniques to prevent further occurrence. Originally, voltage stability studied in a static fashion while the application of machines, exciters, and tap changers is dynamics and can be changed by load flow considerably [7].

Methods that determine the distance of the current operating state from the point of Voltage Collapse (VC) usually express this distance in terms of the additional MVA / MW / MVAR load that the system is capable of supplying before it encounters VC. This additional loading is referred to as Security Margin (SM). In this method, while scheduling the generators for optimum real power outputs the VSM maximization is also taken into account as one of the primary objectives [8].

\section{SCHEDULING FOR ONLINE APPLICATIONS}

As a result of a lot of variables and constraints, the size of the optimization problem becomes large in size and the conventional optimization methods face problems in terms of computational time. Since the computational time has been considerably increased due to problem complexity, the operational engineers face a problem in online scheduling.

Therefore, one can easily see that there is an urgent need to develop powerful methods that can optimize the power system for real power generation in real time. In view of voltage stability issues, it will be beneficial if these newer methods accommodate voltage stability maximization as an objective. Other important security constraints such as line overloading, voltage magnitude limits and others must also be a part of any proposed online real power scheduling scheme. In the following section, OPF optimization techniques are discussed.

\section{OPF OPTIMIZATION TECHNIQUES}

There are many techniques to optimize the power flow which can be classified into different categories as defined in [9]. Nonlinear programming (NLP) is one of the techniques that deals with problems involving nonlinear objectives and constraints. The limitations may involve issues like equality and/or inequality formulations. Several methods such as Sequential Unconstrained Minimization Technique (SUMT), Lagrange multiplier based method can be used to solve OPF problems [10]. These assume nonlinear objectives and constraints.

The Quadratic Programming is a distinctive form of nonlinear programming technique whose main purpose is quadratic and has linear constraints. Quasi-Newton and sensitivity-based methods can be employed for solving real power online OPF problems [11]. Linear Programming (LP) [12, 13] is another commonly applied technique to unravel various linear/nonlinear power system optimization complications like transmission planning, security constrained dispatch, general OPF, emergency control, etc. A set of 
applications known as Variants of simplex based methodologies is commonly employed for resolving the LP complications.

The interior point method (IPM) was developed in [14]. It stunned the operation research community since the scheme solves the linear programming problem much faster than the conventional simplex method. The extension of the interior point method can be applied to NLP and QP problems and has shown superior qualities and promising results. The interior point method, although recently introduced to power system optimization, has developed as a well-suited method and unceasingly modified for many power system optimization glitches. Interior point method for nonlinear programming formulation has been widely applied to answer the OPF issues. This optimization algorithm requires the solution of a set of nonlinear equations to obtain the optimal solution of the power network equations.

In the case of a non-monotonic solution surface, traditional optimization approaches remain very delicate and they often address a local optimum resolution of the system. Hence, there is a need to introduce a new, more general and reliable algorithms that can tackle non-convex solution surfaces. Evolutionary Programming (EP) is one of the main techniques that can be used in this manner [15]. The EP technique is a stochastic optimization method which employs the mechanics of evolution to yield ideal resolution for the problem in hand. This can be done by developing a population of candidate solutions on the way to the overall optimization by employing an evolution operator and a selection scheme. The EP method is predominantly fitted to non-monotonic solution surfaces where many local minima exist.

EP technique was suggested for the development of the finite-state machines in order to solve prediction tasks. There are many adjustments, augmentations, and executions applied and examined. EP technique has been extended to treat real-valued objective functions and other data structures. Transformation is frequently applied by using a new casual number or a vector while using classical EP (CEP) [16] to a parent. The distinction level of the Gaussian mutation is organized using standard deviation or commonly known as "strategy parameter" in the evolutionary search.

The EP algorithm begins by considering a set of possible $\mathrm{P}$ solutions. Let them be referred to as $\mathrm{X} 1$ to $\mathrm{XP}$ where $\mathrm{X} 1$ is the first of such solutions and $\mathrm{XP}$ is the Pth solution. Using this set of $\mathrm{P}$ solutions $\mathrm{X} 1$ to $\mathrm{XP}$, another set of $\mathrm{P}$ solutions $\mathrm{XP}+1$ to $\mathrm{X} 2 \mathrm{P}$ is generated through a random process. From this set of $2 \mathrm{P}$ solutions $\mathrm{X} 1$ to $\mathrm{X} 2 \mathrm{P}, \mathrm{P}$ solutions that satisfy all the constraints and having the best objective function values are chosen. The selected set of $\mathrm{P}$ solutions is hereafter referred to as X1 to XP. With an appropriate termination criterion, it is determined whether the optimum solution has been reached. Otherwise, the set of steps described above is repeated until the optimum is reached.

\section{POWER SYSTEMS STABILITY CLASSIFICATIONS}

\subsection{Rotor Angle Stability}

An interconnected power system is rotor angle stable if it is able to retain synchronism of all its synchronous machines after being subjected to a disruption. The equilibrium between electromagnetic torque and mechanical torque at each synchronous machine in the system must be maintained after the disturbance [17]. When the rotor angle swings of some generators increase and eventually result in their loss of synchronism with other generators, instability occurs. The real power output of a synchronous machine varies proportionally to a function of its rotor angle change. Input mechanical torque and the output electromagnetic torque of a generator balance each other in steady state and its speed remains constant.

On perturbation of the system by a load change, the loading of the generators (electrical torque) changes and hence, they speed up or retard [18]. This change in speed may not be uniform as the change in the load reflects unevenly on the generators. Over time, the system oscillates and finds a new equilibrium wherein all the generators have their mechanical and electrical torques balanced. In certain cases, due to several underlying reasons, these oscillations increase and pull out some machines from synchronism. There are some texts that deal with this problem. The solution schemes for controls have also been researched and cited in

\subsection{Frequency Stability}

The frequency of a power system needs to be maintained at the specified value [19]. Reliability criterion of a power system would restrict the maximum deviation from the set frequency in terms of a number of accumulated cycles of deviation per day. Constantly varying power demand in the power system requires the governor controls to adjust the output of the generators such that they maintain set frequency [20]. However, a rapid change in load or sudden loss of load due to equipment failure would result in frequency excursions. These have to be controlled with a minimum swing in frequency and minimum drop/add of cycles over the entire day. In essence, frequency stability is defined by as "a capability of a power system to uphold steady frequency following a severe system upset resulting in a significant 
imbalance between generation and load." Sustained frequency swings cause loads to be shed or generators cutbacks. These have far-reaching implications on the healthy operation of power systems. Frequency swings alter power flows [21], load profile based upon load characteristics, transmission network matrices and voltages amongst other. It causes undesirable actions such as initiation of voltage/frequency protection termed as over-fluxing protection and others related to boiler dynamics [22]. Large power systems use the frequency as an important criterion for splitting the system into islands for sustained and reliable operation in times of emergency. Frequency stability hence relates to study of the power system while experiencing frequency swings to determine whether the system would be stable taking into considerations a myriad of protective and operational criteria.

\subsection{Voltage Stability}

Voltage stability of a power system reflects its ability to sustain the load by having steady voltages after a disturbance. There are several aspects of the power system that interplay in this issue [23]. These include slow acting voltage control devices like tap setting of transformers, setting of shunt capacitors, setting of shunt reactors and such discrete devices [24]. These also include fast acting devices like generator field excitation control and limiters, static reactive power source controllers, unified power flow controller (its subcategories) and such others [25]. These controllers try to correct the load bus voltages and maintain them within an acceptable range. Some of these also have short-term overload facilities like the generator field current limiters [26]. Due to different time scale operation characteristics of these devices, they may manifest voltage instability. Sometimes, the collapse of a line causes a cascading effect that leads to tripping of other lines and eventual system collapse [27].

Further, while viewing the transmission in Figure 2, there are two conditions in the static view that contributes to voltage collapse. Both of these pertain to lack of reactive power. Thus the two major causes of voltage instability are the lack of reactive power due to high $I^{2} X$ losses in heavily loaded lines and the dynamic nature of the power system. Voltage stability is largely categorized into two types based on its severity.

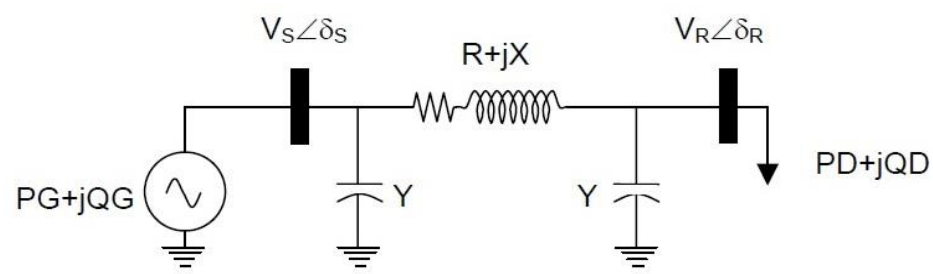

Figure 2. Transmission Line

These characterizations allow the determination of modeling accuracy and type. It also helps in the determination of the kind of simulation required. The first categorization is the large-scale disturbance voltage stability. When the system encounters large disturbances such as loss of generations, symmetric faults, and others, large-scale disturbance voltage stability determines whether the system would retain appropriate voltages at all the buses. This determination requires a non-linear analysis of the power system. It also requires the adequate modeling of slow and fast acting voltage control devices such as tap changers, generator field current limiters, and others. The second characteristic relates to the Small-disturbances and their effects on voltage stability [28]. It tries to assess the structure's capability to keep stable voltages once exposed to minor distresses such as incremental changes in system load. In this case, the characteristics of loads, continuous controls, and discrete controls at a given instant of time influence the response characteristics. Based upon the time frames, assessment methods can be worked out. These time frames and their implications for power system studies are elaborated in the following.

Short-term voltage stability is the first category. This category principally considers the existence of a small time frame for the physical process. All the device models that are simple yet adequately represent the complexities required for the study must be included. Primarily those elements and control actions of the power system that can act in this time frame are considered for modeling. Loads that are varying with respect to the voltage must also be adequately modeled.

Long-term voltage stability includes modeling and adequate representation of slower acting equipment such as tap-changing transformers, thermostatically controlled loads, and generator current limiters. A period of several minutes is required for the long-term simulation to solve these cases. In these 
cases, the interaction of slow acting control like tap changers and generator overexcitation limiters plays an important role. The effect of load recovery after fall of voltage and such characteristics also play defining roles. Their adequate modeling is also of paramount importance. In this paper method of analysis of the voltage stability of the power system is the primary concern. The static measures are adequate as they need to be included in the optimization scheme. Thus, the following section provides statistics in regards to fulfill the research objective.

\section{POWER FLOW JACOBIAN}

In our optimization schemes, there is a need to identify a measure that gives the voltage stability information of the power system. This needs to be related to the control variables of the power system. The singular values of a matrix A are the square roots of the eigenvalues of ATA. Thus, as eigenvalues are adequate indicators of voltage stability, so are the singular values. Hence, in this section, a relationship between singular values of the load flow Jacobian is derived in terms of the states of the power system, namely the bus voltage magnitudes and the bus phase angles.

The incremental change in any singular value of the load flow Jacobian [29] is characterized by the incremental change in the state of the power system. The equations are described in followings. Applying Singular Value Decomposition (SVD), load flow Jacobian, $\left[\mathbf{J}_{\boldsymbol{\delta} V}\right]$ is equated.

$$
\left[\mathrm{J}_{\delta V}\right]=[\mathrm{SU}][\Sigma][\mathrm{SV}] \mathrm{t}
$$

The values of [SU] and [SV] were basically showed orthogonal singular vector matrices; whereas $[\Sigma]$ represents a diagonal matrix containing the singular values. Bearing in mind a minor trepidation in the state, $\Delta \delta$ and $\Delta \mathrm{V}$ can be presented as:

$$
\left[\mathrm{J}_{\delta+\Delta \delta, V+\Delta V}\right]=[\mathrm{SU}+\Delta \mathrm{SU}][\Sigma+\Delta \Sigma][\mathrm{SV}+\Delta \mathrm{SV}]
$$

The LHS of the later equation is extended through Taylor's series. The first order term of the series comprising the load flow Hessian $\left[\mathbf{H}_{\boldsymbol{\delta} V}\right]$ (Load flow Hessian is the first order derivative of Load flow Jacobian) is reserved when ignoring the higher order terms. The following equation is resulted.

$$
\left[\mathrm{J}_{\delta+\Delta \delta, V+\Delta V}\right]-\left[\mathrm{J}_{\delta V}\right]=\left[\mathrm{H}_{\delta V}\right]\left[\begin{array}{c}
\Delta \delta \\
\Delta V_{L}
\end{array}\right]
$$

Using equation (3) to characterize LHS of the equation (2), expanding RHS and preserving only the first order terms, equation(3) is re-written as

$$
\left[\mathbf{H}_{\delta V}\right]\left[\begin{array}{c}
\Delta \boldsymbol{\delta} \\
\Delta \boldsymbol{V}_{\boldsymbol{L}}
\end{array}\right]=[\Delta \mathrm{SU}][\Sigma][\mathrm{SV}] \mathrm{t}+[\mathrm{SU}][\Delta \Sigma][\mathrm{SV}] \mathrm{t}+[\mathrm{SU}][\Sigma][\Delta \mathrm{SV}] \mathrm{t}
$$

Applying orthogonality constraints on the updated left and right singular vector matrices, the following equations are revealed:

$$
\begin{aligned}
& I=[S U+\Delta S U][S U+\Delta S U] t \\
& I=[S V+\Delta S V][S V+\Delta S V] t
\end{aligned}
$$

By expanding the above equations while ignoring the second order terms and using the orthogonality property of [SU] and [SV] have the followings:

$$
\begin{aligned}
& \mathrm{SN}=[\mathrm{SU}] \mathrm{t}[\Delta \mathrm{SU}]=-[\Delta \mathrm{SU}] \mathrm{t}[\mathrm{SU}] \\
& \mathrm{SM}=[\Delta \mathrm{SV}] \mathrm{t}[\mathrm{SV}]=-[\mathrm{SV}] \mathrm{t}[\Delta \mathrm{SV}]
\end{aligned}
$$

According to Venkatesh B., et. al. (2000), the values of [SN] and [SM] as diagonal elements set to be zeros. Pre-multiplying and post-multiplying equation (4) with [SU]t and [SV] respectively, one gets the following: 


$$
[\mathrm{SU}] \mathrm{t}\left[\mathrm{H}_{\boldsymbol{\delta} V}\right]\left[\begin{array}{c}
\boldsymbol{\Delta} \boldsymbol{\Delta} \\
\boldsymbol{\Delta}
\end{array}\right][\boldsymbol{S V}]=[\mathrm{SN}][\Sigma]+[\Delta \Sigma]+[\Sigma][\mathrm{SM}]
$$

Since diagonal elements of $[\mathrm{SN}][\Sigma]$ and $[\Sigma][\mathrm{SM}]$ contain zeros, the diagonal elements of equation (4) are obtained in the following:

$$
\Delta \sigma_{i}=[\Delta \Sigma] \mathrm{ii}=\left[[\mathrm{SU}] \mathrm{t}\left[\mathrm{H}_{\delta V}\right]\left[\begin{array}{c}
\Delta \boldsymbol{\delta} \\
\Delta \boldsymbol{V}_{\boldsymbol{L}}
\end{array}\right][S \boldsymbol{S V}]\right] \mathrm{ii}
$$

Above equation expresses the incremental changes in the singular values of the load flow Jacobian (voltage stability margin in other words), represented by a set of few least singular values of the load flow Jacobian, in terms of the changes in the incremental system variables. The above relation can be gainfully used for optimization methods in power systems. Eigenvalue analysis in relation to the singular values of the load flow Jacobian with the state of the system. These relationships are exploited for maximization of the voltage stability margin. The study offers a practical solution through which proposed technique can adopt different models such as an Artificial Neural Network model [30, 31] for optimal scheduling.

\section{ACKNOWLEDGMENT}

The authors would like to acknowledge the facilities provided by Universiti Teknologi Malaysia for the accomplishment of this work and Ministry of Education (MoE) of Malaysia for their financial support under vote number GUP UTM 17H10. RML is also thankful to NED University of Engineering and Technology Sindh, Pakistan for providing financial assistance by Human Resource Development under the scheme "Strengthening of NED University of Engineering and Technology, Mega-M3" of the Higher Education Commission (HEC), Pakistan.

\section{FUTURE RESEARCH AND CONCLUSIONS}

Optimal power flow techniques are being continuously improved to suit the newly emerging challenges faced by power engineering community. In this pursuit, one or more feature needs to be tackled to improve the voltage stability margin in power systems whereas optimally scheduling power systems. As such, several schemes have been proposed in the literature for the analysis of power system to estimate the voltage stability of a power system. This paper theoretically contributes to developing a new OPF solution method responsive for operational application of power system stability and additional knowledge on the Jacobian model and its application in that essence. Additionally, different optimization techniques have reviewed the aspects with respect to voltage stability. Power system stability classifications are also introduced for developing a promising optimizing scheme for power systems.

The study then presents a statistical method for analyzing a power system through eigenvalue analysis in relation to the singular values of the load flow Jacobian with the state of the system. These relationships are exploited for maximization of the voltage stability margin. The study offers a practical solution through which proposed technique can adopt different models such as an Artificial Neural Network model for optimal scheduling.

Future work may be undertaken in several directions. One of the primary concerns that are evolving in the area of power system optimization is the introduction of market structure. This brings about several changes in theories that were developed, initially for vertically integrated power systems in this research. If generation, transmission, and distribution are governed by different companies, the formulation of the objective function and constraints will become complex and hence further work has to be undertaken to solve the OPF. With regard to the applicability of the proposed scheme to small systems, the online implementation method needs to be tested through new techniques, for example, SCADA (Supervisory control and data acquisition) and PMUs (Phasor Measurement Units) in case of large and complex power systems.

\section{REFERENCES}

[1] D. P. Kothari, "Power system optimization," in Computational Intelligence and Signal Processing (CISP), 2012 2nd National Conference on, 2012, pp. 18-21.

[2] G. Levitin, A. Lisnianski, and D. Elmakis, "Structure optimization of power system with different redundant elements," Electric Power Systems Research, vol. 43, no. 1, pp. 19-27, 1997.

[3] R. M. Larik and M. W. Mustafa, "Smart Grid Technologies in Power Systems: An overview," 2015.

[4] R. M. Larik and M. W. Mustafa, "Technologies used in Smart Grid to implement Power Distribution System," TELKOMNIKA Indonesian Journal of Electrical Engineering, vol. 16, no. 2, pp. 232-237, 2015. 
[5] P. Kundur, N. J. Balu, and M. G. Lauby, Power system stability and control. McGraw-hill New York, 1994.

[6] R. M. Larik, M. W. Mustafa, M. N. Aman, T. A. Jumani, S. Sajid, and M. K. Panjwani, "An Improved Algorithm for Optimal Load Shedding in Power Systems," Energies, vol. 11, no. 7, pp. 1-16, 2018.

[7] G. Subhashini, D. Munandy, and R. Abdulla, "Generating a Lighting System by Using Pico Hydro System," Telkomnika, vol. 15, no. 4, pp. 1565-1573, 2017.

[8] I. R. Mardiyanto, H. Zein, and A. Soeprijanto, "Combining Parameters of Fuel and Greenhouse Gas Costs as Single Objective Function for Optimization of Power Flow," Telkomnika, vol. 15, no. 4, pp. 1585-1600, 2017.

[9] J. A. Momoh, R. Adapa, and M. E. El-Hawary, "A review of selected optimal power flow literature to 1993. I. Nonlinear and quadratic programming approaches," Power Systems, IEEE Transactions on, vol. 14, no. 1, pp. 96104, 1999.

[10] N. M. Saad et al., "Impacts of Photovoltaic Distributed Generation Location and Size on Distribution Power System Network," International Journal of Power Electronics and Drive Systems (IJPEDS), vol. 9, no. 2, pp. 905-913, 2018.

[11] G. M. Rao, "An Adaptive Neuro-Fuzzy Inference Distributed Power Flow Controller (DPFC) In Multi-Machine Power Systems," International Journal of Power Electronics and Drive Systems, vol. 5, no. 2, p. 230, 2014.

[12] W. Shao and V. Vittal, "LP-based OPF for corrective FACTS control to relieve overloads and voltage violations," Power Systems, IEEE Transactions on, vol. 21, no. 4, pp. 1832-1839, 2006.

[13] E. R. Chakravarthy, A. Bhargavi, K. P. Kathirvelu, and R. Balasubramanian, "Analysis and simulation of isolated wind diesel hybrid power system," ARPN J. Eng. Appl. Sci, vol. 9, no. 7, pp. 1056-1063, 2014.

[14] G. L. Torres and V. H. Quintana, "An interior-point method for nonlinear optimal power flow using voltage rectangular coordinates," Power Systems, IEEE Transactions on, vol. 13, no. 4, pp. 1211-1218, 1998.

[15] P. Venkatesh, R. Gnanadass, and N. P. Padhy, "Comparison and application of evolutionary programming techniques to combined economic emission dispatch with line flow constraints," Power Systems, IEEE Transactions on, vol. 18, no. 2, pp. 688-697, 2003.

[16] X. Yao, Y. Liu, and G. Lin, "Evolutionary programming made faster," Evolutionary Computation, IEEE Transactions on, vol. 3, no. 2, pp. 82-102, 1999.

[17] S. P. Singh and S. Singh, "On-line Assessment of Voltage Stability using Synchrophasor Technology," Indonesian Journal of Electrical Engineering and Computer Science, vol. 8, no. 1, pp. 1-8, 2017.

[18] H. Setiadi and K. O. Jones, "Power system design using firefly algorithm for dynamic stability enhancement," Indonesian Journal of Electrical Engineering and Computer Science, vol. 1, no. 3, pp. 446-455, 2016.

[19] J. J. Grainger and W. D. Stevenson, Power system analysis. McGraw-Hill New York, 1994.

[20] D. Gautam, V. Vittal, and T. Harbour, "Impact of increased penetration of DFIG-based wind turbine generators on transient and small signal stability of power systems," Power Systems, IEEE Transactions on, vol. 24, no. 3, pp. 1426-1434, 2009.

[21] R. Singh and R. Mehra, "Qualitative Analysis of Darlington Feedback Amplifier at 45nm Technology," Bulletin of Electrical Engineering and Informatics, vol. 7, no. 1, pp. 21-27, 2018.

[22] P. Venkateswaran, R. Nandi, and S. Das, "Voltage Controlled Integrator and Linear Quadrature-VCO Using MMCC," Bulletin of Electrical Engineering and Informatics, vol. 2, no. 2, pp. 111-116, 2013.

[23] M. K. Debe, A. K. Schmoeckel, G. D. Vernstrom, and R. Atanasoski, "High voltage stability of nanostructured thin film catalysts for PEM fuel cells," Journal of Power Sources, vol. 161, no. 2, pp. 1002-1011, 2006.

[24] S. P. Ong et al., "Voltage, stability and diffusion barrier differences between sodium-ion and lithium-ion intercalation materials," Energy \& Environmental Science, vol. 4, no. 9, pp. 3680-3688, 2011.

[25] M. Lakshmi and R. Kumar, "Optimal Reactive Power Dispatch using Crow search Algorithm," International Journal of Electrical and Computer Engineering (IJECE), vol. 8, no. 3, pp. 1423-1431, 2018.

[26] P. Mohammadi and H. Lesani, "An efficient approach to voltage stability evaluation using Tellegen's equations," International Journal of Electrical and Computer Engineering (IJECE), vol. 3, no. 2, pp. 158-163, 2013.

[27] R. M. Larik, "A New Technique Of Load Shedding To Stabilize Voltage Magnitude And Fast Voltage Stability Index By Using Hybrid Optimization," 2006.

[28] K. V. R. Reddy, M. P. Lalitha, and P. Chennaiah, "Improvement of Voltage Profile through the Optimal Placement of FACTS Using L-Index Method," International Journal of electrical and Computer engineering (IJECE), vol. 4, no. 2, pp. 207-211, 2014.

[29] P. W. Sauer and M. A. Pai, "Power system steady-state stability and the load-flow Jacobian," Power Systems, IEEE Transactions on, vol. 5, no. 4, pp. 1374-1383, 1990.

[30] M. Gevrey, I. Dimopoulos, and S. Lek, "Review and comparison of methods to study the contribution of variables in artificial neural network models," Ecological Modelling, vol. 160, no. 3, pp. 249-264, 2003.

[31] L. Kanagasabai, B. R. Reddy, and M. S. Kalavathi, "Optimal power flow using ant colony search algorithm to evaluate load curtailment incorporating voltage stability margin criterion," International Journal of Electrical and Computer Engineering (IJECE), vol. 3, no. 5, pp. 603-611, 2013. 